\title{
Conservative surgical management of immediate post-caesarean uterine dehiscence and pelvis abscess due to proteus mirabilis infection: a rare complication of puerperal endomyometritis
}

\author{
Chitra Thyagaraju*, Madhuri Makam S., Deepthi Yedla, Dasari Papa
}

Department of Obstetrics and Gynecology, JIPMER, Pondicherry, Tamil Nadu, India

Received: 26 April 2021

Accepted: 28 May 2021

\section{*Correspondence:}

Dr. Chitra Thyagaraju,

E-mail: drchitra@yahoo.com

Copyright: () the author(s), publisher and licensee Medip Academy. This is an open-access article distributed under the terms of the Creative Commons Attribution Non-Commercial License, which permits unrestricted non-commercial use, distribution, and reproduction in any medium, provided the original work is properly cited.

\begin{abstract}
Cesarean delivery is the most commonly performed major abdominal operation in women with prevalence ranging from $12 \%$ in public sectors to $28 \%$ in private sectors in India (DLHS-3 survey). Parallel to this, the complications of surgery are increased. Among these complications, uterine dehiscence and pelvic hematoma with abscess collection is rare but serious complication which might end in hysterectomy. We hereby describe the conservative surgical management of a case of infected uterine incisional necrosis and dehiscence after primary cesarean delivery. We encountered a 25years-old woman presenting to our emergency department (ED) with severe suprapubic pain and high-grade fever. She had an emergency cesarean delivery performed 14 days prior to presentation due to non-reassuring fetal heart rate. At the ED, ultrasonography revealed collection with septation around uterus with communication into uterine cavity. CT scan of pelvis was ordered and showed an intraperitoneal collection anterior to the uterus at the level of the uterine cesarean scar. Exploratory laparotomy showed a uterine rupture at the previous incision site. We performed resection of necrotic edges, peritoneal lavage, approximation of uterine edges with separate interrupted sutures, placement of a suction drain in the cul-de-sac. During postoperative follow up, patient was stable with no symptoms or signs of uterine/pelvic infection. Conservative management by drainage and resection of necrotic edges in addition to intravenous antibiotics may be considered as an option before resorting to hysterectomy in selected young patients.
\end{abstract}

Keywords: Uterine dehiscence, Endomyometritis, Conservative management

\section{INTRODUCTION}

According to data by the Union Ministry of Health and Family Welfare, over 14 per cent of the total births in 2018-19 took place through a C-section. Prevalence of CS births were $13.7 \%$ and $37.9 \%$ in the public and private sectors, respectively. ${ }^{1}$ With the rising caesarean section rates, there is a parallel increase in the cesarean related complications such as post-partum hemorrhage, endomyometritis, localized/generalized peritonitis and sepsis in the short term and placental adhesions disorders, chronic pelvic pain, pelvic adhesion in the long term..$^{2-4}$ The puerperal sepsis/pyrexia presents commonly with fever and other symptoms like pelvic pain, foul smelling vaginal discharge and subinvolution. Out of various causes, septic uterine dehiscence is a rare cause and also difficult to diagnose as patients usually presents with picture of endomyometritis. Incidence of immediate scar dehiscence is rare ranging between 0.06 and 3.8\%. ${ }^{5}$

In this case report, we present a case of a postpartum uterine scar rupture secondary to infection and necrosis which was managed conservatively by drainage and surgical debridement of necrosis with conservation of uterus along with course of broad-spectrum antibiotics.

\section{CASE REPORT}

A 25-years-old female patient was referred from private hospital to our emergency department for high grade fever 
associated with severe pain abdomen of 3 days duration. Patient had undergone emergency LSCS for fetal distress in latent labour, after receiving prophylactic antibiotics at district hospital, 14 days prior to presentation. Postpartum IUCD was inserted before uterine closure and antibiotics was administered for 5 days. Her post-operative period was uneventful and sutures were removed on $8^{\text {th }}$ postoperative day. Her past obstetric history included an uneventful full term normal vaginal delivery.

On presentation, patient was febrile, pulse rate- 112 beats/min and blood pressure 110/70 mmHg. General examination findings were unremarkable. Abdominal examination revealed severe tenderness over suprapubic region with guarding and rigidity and subinvoluted uterus. No distension and scar had healed well with primary intention. Per speculum revealed normal lochia, copper $\mathrm{T}$ was removed under aseptic precautions in an attempt to remove possible source of infection. High vaginal and cervical swab was taken. Laboratory investigations reported $\mathrm{Hb}$ of $8.4 \mathrm{gm} \%$, total count of 8960 cells/cumm with neutrophil predominance $71 \%$ and normal renal and liver function tests. Intravenous $3^{\text {rd }}$ generation Cephalosporin and metronidazole was started empirically. Ultrasonography of abdomen revealed collection of $5 \times 4 \times 3$ with septation near anterior wall of uterus (Figure 1) with suspected rent in uterus.

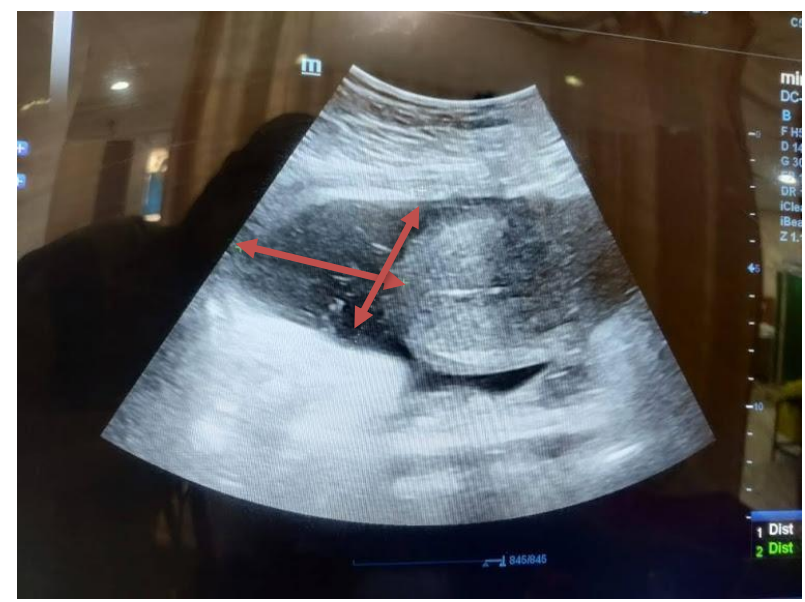

Figure 1: USG of collection around uterus.

Hence, further confirmation with CECT was advised, which revealed a defect in the anterior uterine wall at caesarean scar site with hypodense fluid collection communicating with the collection around the uterus in peritoneal cavity (Figure 2). Since patient condition was deteriorating post 12 hours of IV antibiotics, decision for emergency laparotomy was taken after informed consent.

During exploration, rectus sheath was intact but covered with slough. Around $50 \mathrm{ml}$ of purulent peritoneal fluid was drained, sent for culture sensitivity. Further exploration revealed ruptured uterine scar of $5 \mathrm{~cm}$ with sloughed out margins and inflammatory reaction over anterior surface of uterus (Figure 3).
There was evidence of endometritis and cavity was checked for any products of conception and endometrial swab was sent for culture sensitivity. Uterus as such was not fragile. Given the young age of patient and not so florid sepsis we decided to resuture the incision line and not to perform hysterectomy. Thorough abdominal lavage was ensured, uterine margins were refashioned and defect approximated with interrupted mattress sutures using Polyglactin No.1 suture (Figure 4).

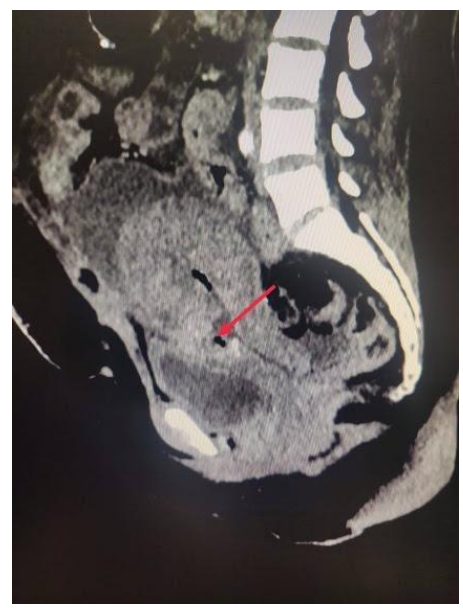

Figure 2: CECT of defect in the anterior uterine wall possibly at the scar site.

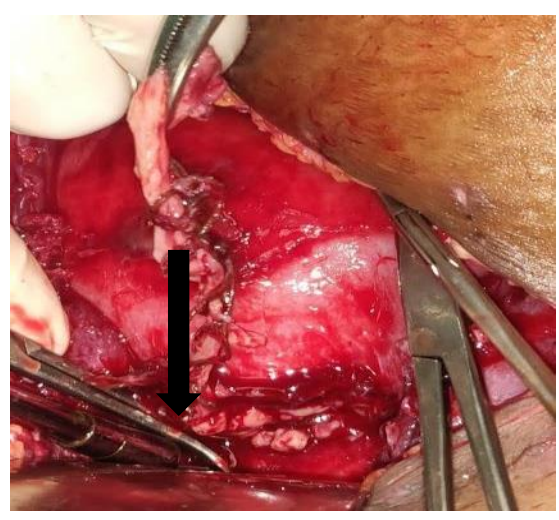

Figure 3: Intra operative- uterine sutures having given away with scar rupture noted.

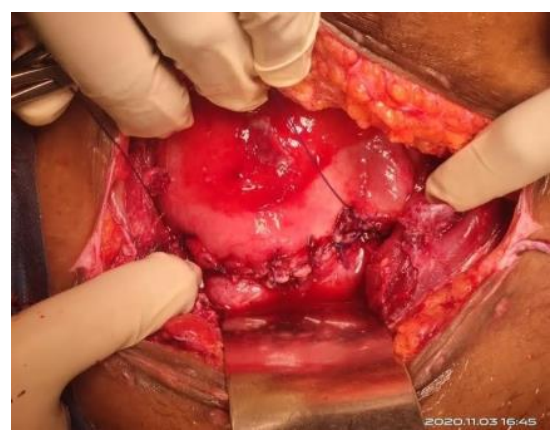

Figure 4: Post debridement, following refreshment of uterine edges, resutured with interrupted Polyglactin No.1 sutures. 
Bilateral tubes were found to be oedematous and ovaries normal. Small bowel loops were distended. Haemostasis was ensured and left pelvic drain was placed. Patient was informed about the conservative management and in case of failure, we would resort to hysterectomy. Post operatively broad spectrum antibiotic meropenem was administered in order to step up antibiotic and continued for 7 days. Post-operative $\mathrm{Hb}$ was $7.4 \mathrm{gm} \%$ and with tachycardia in the operative period, one packed cell was transfused. Subsequently, postoperative recovery was uneventful. Orals was started after 24 hours. Drain and $\mathrm{CBD}$ was removed on third post-operative day.

Table 1: Procedural details.

\begin{tabular}{|llll|}
\hline Date & Pre-operative & POD 1 & POD 3 \\
\hline Hb $($ gm \%) & 8.4 & 7.4 & 8.9 \\
\hline TLC (cells/cumm) & 14,800 & 15470 & 10600 \\
\hline DLC & N71 L20.2 M6.3 & N88.9 L5.8 & N70 120 M6.8 \\
\hline
\end{tabular}

Culture report of high vaginal swab culture grew Proteus mirabilis sensitive to meropenem which she was already receiving. Endometrial swab revealed staphylococcal aureus and enterococcus faecalis sensitive to oxacillin and vancomycin. Since the patient responded well with no further fever spikes or vaginal discharge, no further antibiotic was added. Patient was discharged for follow up on postoperative day 7 in good health after skin suture removal. She was doing well at clinical follow up 3 months after procedure. At 6 months follow up, ultrasound of pelvis showed normal uterine cavity with intact serosa.

\section{DISCUSSION}

The incidence of caesarean section has increased dramatically worldwide especially in developing countries. This has led to increase in complications both short term and long term. puerperal pyrexia is one of the most common complication which contributes to significant preventable maternal morbidity and mortality. It is defined as a sustained temperature of $38^{\circ} \mathrm{C}\left(100.4^{\circ} \mathrm{F}\right)$ or greater beyond the first 24 hours following birth, or a temperature of $39^{\circ} \mathrm{C}\left(102.2^{\circ} \mathrm{F}\right)$ within the first 24 hours following birth (ACOG). Endometritis or endomyometritis wound infection, mastitis, urinary tract infection, and septic thrombophlebitis are the chief causes of puerperal infections. ${ }^{6}$

Uterine dehiscence in the postpartum period due to underlying infection is a rare occurrence but a lethal complication if not diagnosed and treated early. The proposed mechanism is expected to be endomyometritis and infection spreading to the weaker part of uterine wall that is the cesarean incision site leading to uterine scar dehiscence through which blood/pus might have leakage in the surrounding area causing inflammatory reaction and necrosis of the uterine incision site. ${ }^{7}$ Some of the risk factors attributed are diabetes, prolonged labour emergency cesarean section, infection, suture technique, hematoma on the uterine incision line and retrovesical hematoma. ${ }^{8}$

In our case, the risk factors might be emergency LSCS performed at district hospital where experts might not be available during COVID times and the suturing technique or suturing material used as in this Catgut No.1 was used and moreover intraoperative details were not available to rule out any uterine incision extension, haematoma at sutured area etc. Infection associated with uterine scar dehiscence may be present with fever, tachycardia, suprapubic tenderness, uterine tenderness, features of anemia and sepsis. ${ }^{9}$ Puerperal sepsis with localized/generalized peritonitis should raise the suspicion of uterine scar dehiscence/rupture in a case of scarred uterus. To confirm or diagnose uterine dehiscence, imaging techniques such as ultrasonography, magnetic resonance imaging, and computed tomography may be used.

In our case, immediate postoperative status was uneventful and subsequently 2 weeks later developed typical symptoms and signs of peritonitis. Ultrasonography was performed which revealed pelvic collection around uterus with possibility of scar dehiscence. No flow was noted with Doppler applied. CECT abdomen- pelvis being a better diagnostic tool in this condition confirmed a rent in the anterior uterine wall at scar site communicating with the collection in peritoneal cavity. Urgent exploratory laparotomy should be considered with no further delay and decision to conserve the uterus or not depends on the extent of endomyometritis and/or intra-abdominal abscess. Conservative management even in the presence of infection has been reported. ${ }^{10}$

In this case, the culture detected Proteus mirabilis however the wound was surgically repaired with broad spectrum antibiotic cover and there is only one paper reporting Proteus mirabilis as a single agent responsible of this complication, Badr et al where conservative management with uterine debridement and suturing was done with uneventful postoperative period. ${ }^{11}$ Hence, in well-selected patients, debridement and resuture of the uterine incision is an acceptable alternative to hysterectomy for uterine incisional necrosis. Missing diagnosis and delaying treatment may result in septic shock leading to hysterectomy increasing the morbidity and mortality. Hence, an early decision for laparotomy and initiation of broad-spectrum antibiotics is one step favouring conservative management and preservation of fertility. These patients are recommended for repeat cesarean section in the subsequent pregnancy due to high risk of scar rupture. 
Some studies have reported patients presenting with heavy menstrual bleeding after surgery similar to secondary postpartum haemorrhage, so all women who have significant PPH following caesarean should undergo evaluation for any defect in the scar and some even require repair after many years with long term complications such as intermenstrual bleeding and dysmenorrhea or scar rupture in subsequent pregnancy. ${ }^{12}$

\section{CONCLUSION}

Post-cesarean pain in abdomen and puerperal pyrexia needs to be addressed with high suspicion of uterine scar dehiscence even in low-risk women. We should keep in mind the possibility of non-union of the uterine incision leading to secondary postpartum hemorrhage. The main purpose of our report is to improve the current understanding of what constitutes post cesarean scar rupture. Early diagnosis and conservative medical or surgical treatment would prevent aggressive surgical measures such as postpartum hysterectomy with favourably good prognosis thereby decreasing further morbidity and mortality.

Funding: No funding sources

Conflict of interest: None declared

Ethical approval: Not required

\section{REFERENCES}

1. Singh P, Hashmi G, Swain PK. High prevalence of cesarean section births in private sector health facilities- analysis of district level household survey4 (DLHS-4) of India. BMC Public Health. 2018;18(1):613.

2. Wagner MS, Bedard MJ. Postpartum uterine wound dehiscence: a case report. J Obstet Gynaecol Can. 2006;28(8):713-5.

3. Alwani M, Mishra S, Thakur R. Generalized peritonitis with uterine incision necrosis with dehiscence following cesarean section presenting as genitourinary fistula: A unique complication. J Med. 2014;3:762-6.

4. Royo P, Manero MG, Olartecoechea B, Alcazar JL. Two-dimensional power Doppler-three-dimensional ultrasound imaging of a cesarean section dehiscence with utero-peritoneal fistula: a case report. J Med Case Rep. 2009;3:42.

5. Shaamash AH, Ahmed AG, Abdel Latef MM, Abdullah SA. Routine postpartum ultrasonography in the prediction of puerperal uterine complications. Int J Gynaecol Obstet. 2007;98(2):93-9.

6. Maharaj D. Puerperal pyrexia: a review. Part I. Obstet Gynecol Surv. 2007;62(6):393-9.

7. Garg R, Agrawal R, Goel G. Puerperal Sepsis with Uterine Scar Dehiscence after Cesarean Section: A Rare Complication. J South Asian Fed Obstet Gynecol. 2018;10(2):435-7.

8. Agwany AS. Conservative Management of Infected Postpartum Uterine Dehiscence after Cesarean Section. J Med Ultrasound. 2018;26(1):59-61.

9. Bharatam KK, Sivaraja PK, Abineshwar NJ, Thiagarajan V, Thiagarajan DA, Bodduluri S, et al. The tip of the iceberg: Post caesarean wound dehiscence presenting as abdominal wound sepsis. Int J Surg Case Rep. 2015;9:69-71.

10. Rivlin ME, Carroll CS, Morrison JC. Conservative surgery for uterine incisional necrosis complicating cesarean delivery. Obstet Gynecol. 2004;103(5):1105-8.

11. Badr DA, Hassan JM, Ramadan MK. Conservative Surgical Management of Uterine Incisional Necrosis and Dehiscence After Primary Cesarean Delivery Due to Proteus mirabilis Infection: A Case Report and a Review of Literature. J Clin Gynecol Obstet. 2017;6(3):65-70.

12. Sengupta DR, Misra R. Postpartum Uterine Wound Dehiscence Leading to Secondary PPH: Unusual Sequelae. Case Rep Obstet Gynecol. 2012;154685.

Cite this article as: Thyagaraju $\mathrm{C}$, Madhuri MS, Yedla D, Papa D. Conservative surgical management of immediate post-caesarean uterine dehiscence and pelvis abscess due to proteus mirabilis infection: a rare complication of puerperal endomyometritis. Int $\mathbf{J}$ Reprod Contracept Obstet Gynecol 2021;10:2895-8. 